Revue d'histoire de l'Amérique française

DA REVUE D.HISTOIRE DE L'AMÉRIQUE FRANÇAISE

\title{
Théories de la famille et rapports famille-pouvoirs dans le secteur éducatif au Québec et en France (1850-1960)
}

\section{Nadia Fahmy-Eid et Nicole Laurin-Frenette}

Volume 34, numéro 2, septembre 1980

URI : https://id.erudit.org/iderudit/303856ar

DOI : https://doi.org/10.7202/303856ar

Aller au sommaire du numéro

Éditeur(s)

Institut d'histoire de l'Amérique française

ISSN

0035-2357 (imprimé)

1492-1383 (numérique)

Découvrir la revue

Citer cet article

Fahmy-Eid, N. \& Laurin-Frenette, N. (1980). Théories de la famille et rapports famille-pouvoirs dans le secteur éducatif au Québec et en France (1850-1960). Revue d'histoire de l'Amérique française, 34(2), 197-221.

https://doi.org/10.7202/303856ar d'utilisation que vous pouvez consulter en ligne. 


\title{
THÉORIES DE LA FAMILLE ET RAPPORTS FAMILLE-POUVOIRS DANS LE SECTEUR ÉDUCATIF AU QUÉBEC ET EN FRANCE (1850-1960)*
}

\author{
NADIA FAHMY-EID \\ NICOLE LAURIN-FRENETTE \\ Départements d'histoire et de sociologie \\ Université du Québec à Montréal
}

\section{Les théories de la famille}

Les théories de la famille dans les sciences sociales contemporaines se partagent en deux courants principaux: celles qui se rattachent à la conception fonctionnaliste de la société et celles qui se situent dans le cadre du marxisme. Dans la perspective du fonctionnalisme, la famille est définie par ses fonctions ${ }^{1}$. Ces fonctions sont reliées à la satisfaction de besoins humains jugés naturels et universels: la reproduction biologique, le soin et la socialisation des enfants, la satisfaction stable des désirs sexuels et affectifs. La permanence de la famille serait liée à la nécessité et à la persistance de ces besoins. Par ailleurs, selon cette conception, l'évolution des sociétés n'affecte pas la nature de la famille, mais elle affecte la forme de l'institution familiale. En effet, le développement social est conçu par le fonctionnalisme comme un processus de différenciation interne des sociétés que manifeste la spécialisation fonctionnelle de leurs diverses institutions. Ainsi, le développement du capitalisme aurait réduit le nombre des fonctions que la famille doit ellemême exercer en vue d'accomplir sa mission propre. En effet, la production économique s'effectue dans l'usine, l'instruction des jeunes est assumée par l'école, les tâches politiques et policières du

\footnotetext{
* Texte de la Communication présentée au congrès de l'Institut d'histoire de l'Amérique française, à Ottawa, en octobre 1979.

1 À titre d'exemple, voir Talcott Parsons et Robert F. Bales, Family Socialization and Interaction Process (New York, The Free Press, 1955). Aussi, William J. Goode, The Family (Prentice-Hall, Englewood Cliffs, New Jersey, 1964).
} 
chef de famille sont appropriées par l'Etat, etc. Dans la société capitaliste ou bourgeoise, la famille n'exercerait donc spécifiquement que ses fonctions essentielles, les fonctions jugées non essentielles à l'accomplissement de sa mission ayant été prises en charge par d'autres institutions. Cependant, les théories fonctionnalistes s'accordent à reconnaître que certains corrollaires du développement capitaliste ont pour effet d'empêcher la famille et, en particulier, la famille ouvrière, de remplir adéquatement ses fonctions essentielles. La famille devient ainsi dépendante d'autres institutions telles que l'État, l'Église, les organisations d'assistance privées ou publiques, etc. Par ailleurs, les différences et les variations dans l'organisation et le fonctionnement de la famille qui sont liées manifestement aux classes sociales, ne sont jamais rapportées qu'à une différenciation culturelle et, à la limite, morale entre ces classes, ou à des contingences secondaires et temporaires; la famille existe en soi, essence immuable et invariable.

Les théoriciens importants du marxisme ont accordé peu d'attention à la famille et, par conséquent, la théorie marxiste de la famille n'a pas beaucoup progressé au-delà des principes généraux de la problématique de Engels: la division de la société en classes, déterminée par la propriété privée des moyens de production, implique le contrôle des procès sociaux dans l'État et leur reproduction dans la famille ${ }^{2}$. On ne peut négliger, par ailleurs, la contribution de Reich à la théorie marxiste de la famille et celle de l'école de Francfort mais elles s'attachent principalement à l'étude d'une dimension particulière de la famille dans le contexte d'une période historique donnée, à savoir, la formation caractérielle des agents sociaux dans les sociétés fascistes ${ }^{3}$. À tout le moins, on peut affirmer que pour les théories marxistes, la famille n'a pas une nature universelle et permanente. Les formes qu'elle présente et les fonctions qu'elle exerce, dans une période historique donnée et dans une classe sociale quelconque, sont relatives à l'organisation sociale d'un mode de production particulier. La famille s'explique dans son rapport avec les autres appareils de la production, du contrôle et de la reproduction; de même que les autres appareils, la famille s'inscrit dans la

2 Engels, L'origine de la famille, de la propriété privée et de l'État (Paris, Les Éditions sociales).

3 Wilhelm Reich, La psychologie de masse du fascisme (Paris, Payot, 1972). Max Horkheimer, "Authority and the Family», in Critical Theory (New York, Herder and Herder, 1972). 
logique de la production et de la reproduction de l'ensemble des procès sociaux du mode de production.

La recherche marxiste a bien analysé les formes particulières des procès économiques (de production, circulation, etc.) du mode de production capitaliste, mais les procès qui assurent le contrôle et la régulation de la société capitaliste et les procès de sa reproduction, lui demeurent relativement obscurs. Ces procès du mode de production capitaliste et leurs appareils, en particulier la famille et l'État, ont fait l'objet de nombreuses études, mais nous ne disposons pas pour autant d'une théorie universelle de leurs formes et de leur dynamique propres. La plupart des théories relatives à l'organisation politique et idéologique du capitalisme se fondent sur l'étude d'une société particulière ou d'un type particulier de société bourgeoise. Par conséquent, leur champ d'application est limité. Nous croyons qu'il peut être utile de faire ressortir, dans un premier temps, les éléments communs des problématiques les plus courantes touchant l'organisation de la régulation et de la reproduction des sociétés capitalistes et de montrer, dans un second temps, quelles sont les limites du champ d'application de certaines de leurs propositions. La comparaison entre la société québécoise et d'autres sociétés bourgeoises,en particulier la France, permettra d'illustrer certaines particularités des procès de la régulation et de la reproduction d'une société capitaliste, qui ne peuvent être interprétées dans les cadres théoriques existants. Notre démonstration insistera plus particulièrement sur les questions relatives aux appareils qui sont considérés comme les plus importants du point de vue de la régulation et de la reproduction: l'État, l'Église et la famille.

Il est généralement admis que ces appareils de la régulation et de la reproduction des sociétés capitalistes exercent deux catégories principales de fonctions. D'une part, ils établissent et garantissent diverses conditions techniques, économiques et juridiques de la production: aménagement du territoire, règlementation des échanges, etc. D'autre part, ils assurent le contrôle des populations. Cette fonction est multidimensionnelle et comprend aussi bien le contrôle démographique de la population et le contrôle de ses déplacements dans l'espace que l'éducation, l'assistance et la police. La plupart des théories considèrent que l'État occupe, en général, une place centrale dans les réseaux des appareils qui exercent l'ensemble de ces fonctions. On considère que l'organisation des fonctions techniques, économiques et juridiques de la régulation et de la reproduction, repose sur une articulation complexe de l'État aux appareils 
économiques des secteurs de la finance, de l'industrie, du commerce, du travail et autres. L'organisation du contrôle des populations impliquerait, par ailleurs, une articulation également complexe de l'État aux appareils culturels, religieux, scolaires, médicaux, etc., et, en particulier, à la famille. Pour l'école structuraliste d'inspiration althussérienne, qui a fourni le cadre d'une substantielle reformulation de la théorie marxiste au cours des dernières décennies, l'État représente le centre moteur du système de régulation-répression de la société capitaliste. Les mécanismes-clé de ce système sont qualifiés d'appareils idéologiques d'État (A.I.E.) parce qu'ils «fonctionnent» à l'idéologie comme l'écrit Althusser, et qu'ils sont soumis plus ou moins complètement à la stratégie étatique. Ainsi, la famille, l'école, l'Église, les media d'information et les diverses institutions de la culture sont rangés dans cette catégorie très hétéroclite des appareils idéologiques d'État ${ }^{4}$. En ce qui concerne la famille, il faut signaler qu'il s'agit essentiellement d'une position de principe car la nature de son statut d'A.I.E. et son mode d'insertion dans le système général des A.I.E. ne sont explicités dans aucun des travaux d'inspiration althussérienne. De même pour l'Église, dont l'école althussérienne n'a défini et illustré nulle part la nature et le fonctionnement en tant que A.I.E. En revanche, d'autres courants théoriques récents, qui s'inspirent du marxisme jusqu'à un certain point et dont on peut situer les travaux à la jonction de l'histoire et de la sociologie, ont présenté la famille comme l'élément-clé, la charnière de l'organisation du contrôle multidimensionnel des populations, propre aux sociétés bourgeoises ${ }^{5}$.

Ces théories et ces travaux de recherche considèrent en général que la famille est tout à la fois la cible et le moyen des multiples formes du contrôle social. La famille serait ainsi l'objet privilégié de ce qu'on appelle depuis quelques années, les stratégies du pouvoir. Selon ces théories, les formes modernes de la famille sont déterminées par l'intégration de l'appareil familial dans les réseaux de la régulation et de la reproduction centrés dans l'État bourgeois.

4 Voir Althusser, «Idéologie et appareils idéologiques d’État», La Pensée (avril 1970).

Voir, en particulier, les ouvrages suivants: Michel Foucauld, La volonté de savoir (Paris, Gallimard, 1976) et Surveiller et punir (Paris, Gallimard, 1975). En collaboration: Isaac Joseph, Philippe Fritsch et Alain Battegay, «Disciplines à domicile, l'édification de la famille», Recherches, no 28 (1977). Jacques Donzelot, La police des familles (Paris, Éditions de Minuit, 1977). Philippe Meyer, L'enfant et la raison d'Etat (Paris, Editions du Seuil, Collection Points, 1977). Voir aussi les communications présentées au colloque «Famille et pouvoirs», Aix-en-Provence (juin 1979), à paraître. 
Le processus de constitution de la famille moderne dans et par l'État, se serait amorcé vers le 17e siècle; il aurait impliqué la dissolution ou la transformation des formes familiales antérieures à la société bourgeoise. Diverses interventions, qualifiées de normalisatrices, touchant la vie privée des individus, auraient permis d'accomplir progressivement cette transformation. Ces interventions «normalisatrices» auraient été générées par diverses institutions encadrées et coordonnées par l'État: les institutions scolaires, les institutions de patronage et d'assistance publiques et privées, l'institution médicale, les Églises, etc. Ainsi, vers la fin du $19 \mathrm{e}$ siècle, le modèle de la famille bourgeoise se trouverait solidement implanté dans l'ensemble de la société et représenterait une des garanties essentielles de prospérité et d'ordre social, d'une part, parce qu'il assure aux classes privilégiées la reproduction de leur capital en biens et en savoir; d'autre part, parce qu'il permet de fixer, diviser et encadrer les classes populaires.

\section{Diversité des sociétés bourgeoises}

À notre avis, les théories fonctionnalistes de la famille sont peu utiles à l'étude de la famille dans la société capitaliste. Elles permettent difficilement de rendre compte, entre autres, des conditions et du processus de constitution de la famille et de son intégration dans les réseaux du contrôle et de la reproduction de cette société capitaliste. En effet, ces théories postulent une nature humaine intangible et immuable dont dériveraient les fonctions des institutions sociales et elles réduisent le développement des sociétés à l'adaptation des formes sociales à ces fonctions. Par contre, les théories et les travaux sur la famille qui s'inspirent d'une conception marxiste de la société, nous semblent plus intéressants. En effet, il faut admettre à l'instar de leurs auteurs que le résultat universel de l'organisation, de la régulation et de la reproduction dans la société capitaliste, est bien l'intégration de la famille dans le réseau des appareils du pouvoir et que cette intégration fait de la famille un des mécanismes privilégiés de contrôle des populations. Cependant, il nous semble que le processus social qui détermine historiquement cette intégration de la famille aux appareils du pouvoir n'est pas le même dans toutes les sociétés capitalistes. De même, il nous semble que les modalités de cette intégration de la famille aux appareils du pouvoir, dans les sociétés capitalistes, présentent une importante diversité. Or, les théories actuelles de la famille, dérivées du marxisme, ne permettent pas de rendre compte de cette 
variabilité. Les travaux qui rendent compte de la transformation de la famille en mécanisme du pouvoir, s'inspirent des formes historiques que ce processus a revêtues dans certaines sociétés de l'Europe occidentale et, en particulier, dans la société française. Ces recherches ne tiennent pas compte cependant, des particularités qui ont marqué le développement de l'organisation économique, politique et idéologique du capitalisme dans ces sociétés et qui ont affecté, par là, l'évolution des formes familiales. Deux éléments, en particulier, ont déterminé, selon nous, le processus et le mode d'intégration spécifiques de la famille aux réseaux du pouvoir, dans ces sociétés. En premier lieu, la forme centralisée et bureaucratique de l'État bourgeois, qui découle de ses formes et de son évolution antérieures et qui doit être associée à la constitution des corps de fonctionnaires étatiques et para-étatiques responsables des interventions «normalisatrices» auprès de la famille: enseignants, assistants sociaux, hygiénistes, médecins, juristes, scientifiques, etc. En second lieu, l'absence d'une alliance étroite et permanente entre les fractions de la classe dominante liées d'une part à l'Église et, d'autre part, à l'État et aux diverses bases de la propriété.

Le développement du capitalisme dans d'autres sociétés ne présente pas ces deux particularités. Il en découle, en particulier, que le processus et le mode d'imbrication de la famille dans les réseaux du pouvoir, sont différents de ceux qu'on peut observer ailleurs, surtout dans la France du 17 e au 20e siècle. Or, c'est le cas de la famille française et d'autres cas analogues que les travaux récents d'histoire et de sociologie de la famille ont eu tendance à généraliser et à théoriser. Aux États-Unis et au Canada, l'État bourgeois prend une forme décentralisée et la bureaucratie étatique et para-étatique connaît un faible développement jusqu'au milieu du 20e siècle. Aux Etats-Unis, les interventions «normalisatrices» auprès de la famille s'effectuent dans une large mesure hors de l'État, même si ce dernier en fournit parfois le cadre juridique. Encore faut-il signaler qu'il ne s'agit pas le plus souvent de l'État central mais des gouvernements locaux, ceux des municipalités et des États, dont l'action, en outre, est rarement concertée. On remarque, entre autres, que la famille américaine est impliquée dans des réseaux d'organisations civiques concurrentes, dites associations volontaires, qui regroupent les citoyens sur la base de leur âge, race, ethnie, sexe, classe, profession, religion, etc. ${ }^{6}$. Ces associations volontaires sont

6 Voir entre autres références, Michael Gordon, ed., The American family in socialhistorical perspective (New York, St.Martin's Press, 1973). 
autonomes et relèvent entierement de l'initiative privée contrairement aux associations de patronage françaises, par exemple, qui sont encadrées par l'État et, souvent, en dépendent. En outre, l'apport de l'immigration à la reproduction de la main-d'oeuvre rend superflu le contrôle démographique de la population par l'État. Or, on sait qu'un tel contrôle est en partie responsable ailleurs, en France par exemple, de l'investissement de la famille par l'État. En Angleterre, l'État pratique le laisser-faire démographique mais dans un contexte différent de celui des États-Unis. Historiquement, il est probable que les politiques de l'État britannique affectent la famille surtout dans ses conditions matérielles d'existence, par l'intermédiaire des lois fixant les modalités du travail des femmes et des enfants et par l'assistance financière sous la forme des prestations sociales ${ }^{7}$.

Par ailleurs, dans plusieurs sociétés capitalistes, notamment en Italie, en Espagne et au Québec, l'Église joue un rôle privilégié dans le développement de l'organisation sociale bourgeoise. L'Église y est intégrée de façon organique dans la classe dominante, intégration que traduit une division stable et efficace du pouvoir entre l'Église et l'État. On peut d'ailleurs penser que la faible articulation interne de la classe dominante (c'est-à-dire de ses composantes économiques et régionales), rend possible sinon nécessaire, dans ces sociétés, le partage du pouvoir entre l'Église et l'État. Or, ce partage soustrait dans une large mesure la famille au contrôle de l'État. C'est principalement l'Eglise qui organise alors la famille comme mécanisme de régulation et de reproduction de la société capitaliste. Les limites de l'intervention de l'État auprès de la famille sont fixées par l'Église, de manière implicite comme au Québec ou de manière explicite comme dans l'Italie du Concordat ${ }^{8}$. L'État doit ainsi tenir compte, dans le mode, l'objet et la fréquence de ses interventions, de cette dominance de l'Église dans le champ de la famille. En outre, cet investissement de la famille par l'Église affecte la forme de l'organisation familiale, en particulier les rapports entre ses membres. En effet, diverses observations permettent d'envisager l'hypothèse selon laquelle l'Église exercerait son

Pour l'Angleterre, voir Mary Mclntosh, «The state and the oppression of women», in A. Kuhn et A.M. Wolpe, eds., Feminism and Materialism (London, Routledge and Kegan Paul, 1978).

8 Pour l'Italie, voir Lesley Caldwell, «Church, state and family: the women's movement in Italy", in Feminism and Materialism, op. cit. et pour la période du fascisme, Maria A. Macciocchi, Éléments pour une analyse du fascisme (Paris, Collection 10/18, 1976). 
influence sur la famille principalement par l'intermédiaire des femmes, contrairement à l'État qui utiliserait plutôt les hommes comme courroie de transmission.

L'intervention de l'Église auprès de la famille utilise un éventail de moyens et de techniques traditionnels qu'on peut énumérer rapidement car ils nous sont connus. Dans les sociétés où elle détient une place dominante dans le réseau des appareils du contrôle, l'Église dispose souvent d'un contrôle partiel ou exclusif de l'enregistrement de la population, ce qui lui permet de connaître et de surveiller les divers mouvements socio-démographiques de cette population: naissances, mariages, décès, etc. Les groupes religieux, les associations et les confréries religieuses assurent l'encadrement (l'enrégimentation) de toutes les catégories de la population, divisée selon le sexe, l'âge, la profession, etc. Les mécanismes de l'inculcation idéologique sont mis en oeuvre dans l'appareil scolaire confessionnel et par la prédication et les autres modes de diffusion du discours religieux. Il faut ajouter à cette liste, les divers moyens «personnels» de surveillance et d'aveu dont l'Église dispose: confession, visite des familles, etc. Enfin, on doit aussi signaler les techniques de «normalisation» des déviants et des marginaux de toutes sortes, qui s'associent aux pratiques de l'assistance aux pauvres, aux orphelins et autres. Dans la période contemporaine, l'Église a continué d'utiliser ces moyens et ces techniques traditionnels mais en les adaptant aux nouvelles conditions de vie de la population et, en particulier, à celles des classes populaires urbaines. Ainsi, le système scolaire, le système hospitalier et celui de l'assistance sociale en particulier, placés sous le contrôle du clergé et des communautés religieuses, ont été étendus à une échelle plus vaste et leur gestion a été modernisée. De même, le réseau des associations et des groupements religieux et para-religieux qui assure l'encadrement de la population, a été diversifié et étendu aux dimensions nouvelles de l'existence comme la pratique syndicale, le loisir, etc.

Comme nous l'avons remarqué précédemment, l'investissement de la famille par l'Église, dans le contexte d'une société capitaliste, n'est pas un phénomène propre au Québec. Cependant, le mode d'articulation de l'appareil ecclésial et de l'appareil étatique dans cette société, en fait un cas original, qui va peut-être plus loin, selon nous, que l'influence très grande que l'Église a exercée surr l'État en Italie, en Espagne et ailleurs. En effet, les deux appareils se sont trouvés pratiquement imbriqués l'un dans l'autre non seule- 
ment à leur sommet mais à tous les paliers de leur hiérarchie respective au point de s'unir quasiment en un seul appareil. Ce qui, sans exclure la possibilité de conflits entre l'Église et l'État, leur a permis d'exercer ensemble une forme de pouvoir qu'on peut qualifier de totalitaire. Nous pensons que le caractère singulier de ce mode d'exercice du pouvoir ressort avec évidence de la comparaison historique entre les systèmes scolaires québécois et français, que nous présentons dans la prochaine section.

En ce qui concerne la France, il faut d'ailleurs remarquer que les travaux récents ont peut-être sous-estimé l'importance et surtout l'autonomie de l'Église dans le champ familial. En effet, l'État français n'est pas toujours parvenu à encadrer et à gérer tous les modes d'intervention de l'appareil ecclésial auprès de la famille. Il semble, à cet égard, que l'Église a pu tirer profit de la division de la bourgeoisie française sur la question de la religion et de ses rapports avec la politique. Les données historiques que nous analyserons plus loin illustrent certaines conséquences de cette situation, par exemple, l'éducation confessionnelle des enfants de nombreuses familles bourgeoises et la prédominance de l'Église dans le système d'éducation des filles. De même, dans le cas des États-Unis, on peut être porté à négliger l'articulation de la famille aux Églises. Des travaux récents semblent indiquer que cette articulation a revêtu une forme originale et qu'elle a entraîné des conséquences culturelles jusqu'ici insoupçonnées. En effet, écartés du pouvoir politique et de l'activité économique, au début du 19e siècle, les ministres des dénominations protestantes les plus influentes et les femmes de la bourgeoisie auraient formé une alliance stable, fondée sur la dépendance réciproque, alliance qui leur aurait assuré le contrôle direct ou indirect d'une large part du champ de la culture (au sens large) via la production et la diffusion d'une idéologie religieuse et familialiste basée sur le «sentiment» ${ }^{9}$.

Il faut remarquer que le cas des sociétés précédemment citées met en cause non seulement les théories de la famille comme appareil d'État, mais aussi la définition et l'utilisation courantes de la notion de mode de production. En effet, nombre de chercheurs conçoivent le mode de production à la manière d'un modèle formel et idéal, dont l'application permet l'analyse et la classification des sociétés «concrètes». Dans cette perspective, un mode de produc-

9 Telle est la thèse démontrée par Ann Douglas, dans The Feminization of American culture (New York, Avon Books, 1978). 
tion est une combinatoire spécifique de procès, de classes et d'appareils particuliers. Ainsi, les combinatoires servage/pouvoir féodal/ Église ou encore, salariat/État libéral/appareil idéologique bourgeois (laïque). Lorsqu'une société ne correspond pas au modèle, elle est traitée comme une exception, un écart par rapport à la norme. Ainsi, les sociétés féodales où l'État prend une forme non absolutiste sont jugées «en avance» et les sociétés capitalistes où l'Église exerce des fonctions importantes sont jugées «en retard». À ce jeu, une bonne part de la réalité devient une exception en regard de la théorie, ce qui évite de reconnaître et de théoriser la variété et la complexité de l'organisation sociale d'un mode de production. Le peu d'intérêt théorique et empirique que les travaux marxistes, en particulier, ont accordé à l'analyse de la place et des fonctions de l'Église catholique (et des autres églises) dans la société capitaliste, est une des conséquences particulièrement importantes de cette conception erronée du mode de production.

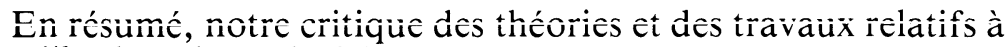
la famille dans la société bourgeoise s'appuie sur l'hypothèse de l'interaction et de la complémentarité des appareils politiques et religieux dans la régulation et le contrôle de l'organisation sociale du capitalisme. Et ce, d'autant que cette régulation et ce contrôle s'exercent dans la vie dite privée, par l'intermédiaire des divers systèmes de pratiques qui constituent la famille, la surveillent, la soutiennent et la reproduisent d'une part et qui, d'autre part, s'appuient sur la famille, en dépendent et l'utilisent. Le système scolaire et celui de l'assistance sociale, en particulier, fournissent le cadre de ces modes d'intervention. Toutefois, c'est probablement l'école qui représente le lieu privilégié de ces réseaux complexes de relations réciproques entre la famille et les appareils de la régulation et du contrôle, relations qui créent et reproduisent la distinction entre l'ordre de la vie privée et celui de la vie publique et qui assurent l'articulation fonctionnelle de ces deux secteurs. L'illustration satisfaisante de notre hypothèse exigerait que l'on établisse une comparaison systématique entre les sociétés bourgeoises, prises à un stade semblable de leur développement. Cette comparaison mettrait en lumière le rôle respectif de l'appareil religieux et de l'appareil politique dans la formation et la conservation de l'organisation familiale caractéristique de la société bourgeoise. Nous pensons qu'on arriverait ainsi à délimiter trois types de société: celles où la famille s'articule principalement à l'Église, celles où elle s'articule principalement à l'État et celles où la famille s'articule autant à l'un qu'à l'au- 
tre appareil. L'étude de secteurs-clé, d'abord le système scolaire et aussi celui de l'assistance sociale, (aussi bien de leur structure que du contenu de leurs interventions), permettrait de reconstituer ces modes d'articulation. La comparaison devrait ensuite être étendue à d'autres dimensions de l'organisation économique et sociale de ces sociétés, de manière à cerner la spécificité de chacun des trois types ci-haut mentionnés, en ce qui concerne par exemple, les particularités du développement capitaliste, la forme du système politique, les rapports entre les classes et les fractions de classe, la culture, etc.

Dans le cadre de cet article, nous nous sommes limitées à une comparaison entre la France et le Québec, qu'illustre une mise en parallèle du développement et du mode de fonctionnement de leur système scolaire respectif. Ces deux sociétés représentent des cas exemplaires jusqu'à un certain point, en ce qui concerne l'articulation famille-Église-État. En outre, elles ont fait l'objet d'études qui nous sont mieux connues que celles qui portent sur d'autres sociétés. Cette comparaison est susceptible de donner un caractère plus concret à notre critique des théories et des travaux sur la famille et d'éclairer partiellement les hypothèses que nous avons avancées, mais elle ne peut suffire à les fonder entièrement. Aussi, souhaitons-nous ouvrir la voie à des études comparatives du même genre qui permettraient d'approfondir la démarche entreprise dans le présent travail.

Dans la section subséquente, nous procéderons à une comparaison du mode d'intégration de la famille à l'Église et à l'État, au Québec et en France, à partir de diverses données touchant l'éducation et le système scolaire, qui feront l'objet d'une analyse systématique $^{9 \mathrm{a}}$. Au terme de cette comparaison, nous évoquerons la prise en charge exclusive et universelle de la famille par l'État au cours des dernières décennies, ce qui nous permettra d'ajouter d'autres remarques et interrogations théoriques et historiques à celles qui nous ont servi de point de départ.

9a Nous aurions souhaité procéder à une étude systématique de données équivalentes touchant le système de l'assistance sociale dans les deux sociétés. Toutefois, les exigences méthodologiques de la démarche d'analyse historique comparative de matériel empirique, rendaient cette tâche très laborieuse et auraient allongé exagérément le texte de cet article. 


\section{LES RAPPORTS FAMILLE-ÉGLISE-ÉTAT DANS LE SEC- TEUR ÉDUCATIF: LES CAS DU QUÉBEC ET DE LA FRANCE, 1850-1960}

La perspective comparative que nous adopterons ici se limitera à explorer un domaine précis où l'évolution des rapports entre la famille et le pouvoir civil et religieux dans les deux sociétés française et québécoise nous est apparu particulièrement riche de sens ${ }^{10}$. Il s'agit plus précisément du secteur de l'éducation. Dans ce domaine, notre démarche et les interrogations qui s'y rapportent se situeront dans le cadre d'une configuration triangulaire où l'on retrouvera à un pôle la famille ${ }^{11}$, et aux deux autres le pouvoir étatique et le pouvoir religieux.

Pourquoi cependant avoir choisi la société française et ses institutions comme point de comparaison? Nous croyons de fait, que la société française, autant que la société américaine d'ailleurs ${ }^{12}$, a constitué un pôle de référence majeur vers lequel se sont fréquemment tournés les individus et les institutions au Québec. Ceci aussi bien, faut-il le préciser, pour s'en inspirer que pour affirmer vouloir s'en démarquer.

Une dernière mise au point, d'ordre méthodologique, s'impose enfin: devant obligatoirement schématiser notre exposé pour éviter de l'étaler indûment, nous avons préféré le faire parfois aux dépens de détails relatifs à l'histoire québécoise, surtout lorsque nous avions quelques raisons de croire que ces détails étaient plutôt familiers à une majorité de lecteurs d'ici. Les tableaux socio-historiques comparés qui figurent dans notre étude comportent donc le plus souvent, pour un secteur déterminé, moins de données relatives à la société québécoise qu'à la société française.

10 D'autres sujets cependant, tels que «Dimensions de la famille et pouvoirs», "Droit familial et pouvoirs», "Assistance sociale aux familles et pouvoirs», font partie d'une série d'interrogations où l'analyse comparée entre les sociétés française et québécoise, au tournant du siècle en particulier, pourrait s'avérer très fructueuse. Ce ne sont là que des exemples, parmi d'autres, de champs de recherche encore peu explorés par l'historiographie au Québec.

11 Il faudrait préciser, à ce niveau, qu'il s'agit moins, dans notre perspective, de l'institution familiale comme telle que de la famille comme cellule constitutive d'un groupe social donné. On devrait dans cette optique parler autant, sinon plus, des familles (au pluriel) que de la famille.

12 Il aurait été effectivement intéressant d'établir une analyse historique comparative en choisissant cette fois, comme pôle de comparaison, la société américaine du milieu du $19 \mathrm{e}$ siècle. Nous n'avons pu entreprendre une telle analyse nous-mêmes, vu les dimensions déjà très vastes du sujet que nous traitons ici. 
Il sera question surtout de l'encadrement civil et religieux de l'enfant et de la famille, par le biais de l'institution scolaire. Nous postulons tout d'abord que, lorsqu'il s'agit de rapports de pouvoir, et non d'apprentissage comme tel, la famille est aussi concernée que l'enfant. Nous avons pu constater par ailleurs qu'à travers la rivalité qu'entretiennent les institutions et les luttes qui opposent entre eux les groupes sociaux en présence, se profilent constamment un discours et une pratique qui s'adressent en grande partie aux familles, implicitement ou explicitement reconnues comme principales interlocutrices dans toutes les questions relatives à l'éducation.

Quand on compare l'évolution du système éducatif français et québécois, surtout au cours de la seconde moitié du 19e siècle, ce qui retient l'attention, de prime abord, ce sont les contrastes que présentent les deux systèmes à maints niveaux, différences qui n'excluent nullement - nous le verrons plus loin - des analogies à la fois intéressantes et fort significatives.

\section{Les contrastes}

Au Québec, le système scolaire a constitué, depuis sa mise en place en 1841 et jusqu'à la Révolution tranquille, un champ du social où l'encadrement effectif de l'enfant et de la famille par l'Église ne trouve son équivalent que dans peu de sociétés occidentales au cours de la même époque ${ }^{13}$. On sait jusqu'à quel point $y$ fut poussé le degré de confessionnalisation du système éducatif tant au niveau primaire qu'aux niveaux secondaire, collégial et même universitaire ${ }^{14}$.

Durant plus d'un siècle, les familles québécoises ont pris conscience qu'en ce qui concernait l'éducation de leurs enfants, elles devaient compter à la fois avec l'Église et sur elle. Ceci tant en ce qui

13 Il faut se tourner vers l'histoire sociale de l'Irlande ou de l'Italie pour trouver des analogies - étonnantes d'ailleurs - avec l'histoire québécoise en ce qui a trait à l'étendue et à la pérennité de l'influence cléricale dans le champ du social.

14 Nous référerons le lecteur à deux synthèses, considérées presque comme des classiques, dans le domaine de l'histoire de l'éducation au Québec. Il s'agit des travaux de LouisPhilippe Audet, résumés dans une synthèse intitulée: Histoire de l'enseignement au Québec (Montréal, HRW, 1971, 2 vol.) et, une quarantaine d'années plus tôt, le tableau panoramique brossé par Lionel Groulx, dans L'enseignement français au Canada (Montréal, 1931 et 1935,2 vol.). En ce qui a trait à l'enseignement collégial, il y a également l'ouvrage récent de l'historien Claude Galarneau sur Les collèges classiques au Canada français (Montréal, Fides, 1978). 
avait trait à la structure et au fonctionnement du système scolaire qu'en ce qui regardait son orientation pédagogique et morale. L'État, quant à lui, a assumé surtout le rôle de pourvoyeur. Il constituait une source de financement et un agent administratif, indispensable certes, mais soumis toutefois aux directives émanant de la hiérarchie religieuse. Jusqu'au milieu du 20 e siècle, le discours clérical sur la famille et l'éducation reflète d'ailleurs assez fidèlement la structure des rapports de pouvoir qui prévalaient au sein de l'univers éducatif. De plus, ce discours traduit la perspective des autorités religieuses et politiques relativement au rôle dévolu à l'institution familiale dans le cadre de ces rapports. On se rend compte alors que la famille est considérée comme le pivot central autour duquel s'élaborent l'ensemble des stratégies liées aux politiques éducatives. Elle constitue un partenaire qu'il faut s'adjoindre puisque c'est par lui que passent nécessairement les axes des trajectoires poursuivies par ces politiques ${ }^{15}$. Or, durant plus d'un siècle, le discours idéologique dominant a présenté la famille comme l'alliée naturelle de i'Égiise face à un État constamment suspect de vouloir déborder le cadre de ses compétences en matière d'éducation.

Au Québec, la pratique autant que le discours éducatif ont créé et maintenu ainsi une tradition d'alliance entre la famille et l'Église, contribuant par le fait même à contenir le pouvoir étatique dans des frontières aux tracés bien définis et surtout peu mobiles.

En France, à partir du milieu du 19e siècle, les rapports de pouvoir dans le champ de l'éducation ont connu une évolution manifestement différente de celle qui a caractérisé ces mêmes rapports dans la société québécoise à la même époque ${ }^{16}$. On sait à quel

15 Certains ouvrages, qui s'étalent tout au long de la période considérée (1850-1950), illustrent de façon éloquente la thèse mentionnée ci-haut. Cf. L. Laflèche, Quelques considérations sur les rapports de la société civile avec la religion et la famille (Montréal, 1866). A. Mailloux, Manuel des parents chrétiens (Québec, 1851). L'éducation ou la grande question sociale du jour. Recueil de documents (Montréal 1886), (s.n.d.). Au 20e siècle, de multiples articles parus dans les Semaines Sociales du Canada continuent à désigner la famille comme l'alliée indispensable de l'Église parce qu'étant l'élément-clé de toute politique éducative. Voir en particulier à ce sujet les comptes rendus des Semaines Sociales publiées à partir des thèmes suivants: La Famille (1923), Le Chrétien dans la famille et la nation (1940), Le Foyer base de la société (1950). L'abbé Lionel Groulx adopte un point de vue analogue lorsqu'il traite de «La Famille canadienne-française, ses traditions, son rôle», in Notre Maître le Passé (Montréal, 1924), 101-135. Il en va de même pour Mgr L.-A. Paquet dans certains de ses écrits réunis sous le titre: Études et appréciations. Nouveaux Mélanges canadiens (Québec, 1919).

16 Voir à ce sujet les deux remarquables synthèses d'A. Prost: Histoire de l'enseignement en France (A. Colin, coll. II, 1968) et de M. Crubelier, L'enfance et la jeunesse dans la société française, 1800-1950 (A. Colin, 1979); il y a également le résumé succinct d'A. Léon, Histoire de l'enseignement en France (Paris, P.U.F. 1967). 
point - contrairement à ce qui a prévalu au Québec - les relations entre l'Église et l'État y furent marquées par un affrontement qui a revêtu une forme tantôt ouverte, tantôt larvée, un affrontement qui demeura constant pendant plus d'un demi-siècle, même s'il fut ponctué parfois de quelques trêves ${ }^{17}$. Il s'agissait de fait, pour chacun des deux pouvoirs (étatique et religieux) de s'assurer le contrôle effectif, sinon exclusif, d'un champ du social où il constituerait le seul interlocuteur valable auprès des enfants et des familles évoluant dans son orbite.

La rivalité entre l'Église et l'État s'est exprimée à travers des modalités et aussi des résultats différents selon le niveau pédagogique concerné. Ce dernier commandait en effet une stratégie politique particulière dans la mesure où, derrière chaque niveau - élémentaire, secondaire et universitaire - se profilaient des groupes sociaux différents. Le rapport Famille/État/Église variera donc à la fois dans sa forme et dans son évolution selon les niveaux d'enseignements et l'origine sociale des familles concernées.

En ce qui a trait à l'école élémentaire, on constate que l'Église a dû, dans un laps de temps relativement court, céder à l'État une grande partie de son champ d'influence dans ce domaine ${ }^{18}$. C'est ainsi que, dès le milieu du 19e siècle, l'État en France n'a pas cessé de consolider son emprise sur ce que l'historien français de l'éducation, Antoine Prost, appelle «l'école du peuple» (l'école élémentaire publique). L'État l'a fait, bien entendu, au détriment du pouvoir religieux qui était jusque-là l'interlocuteur principal, en matière d'éducation, auprès des enfants et des familles des classes populaires. Il l'a fait même au détriment de congrégations religieuses bien implantées dans le domaine de l'éducation comme l'étaient les Frères des Écoles Chrétiennes.

Contrairement aussi à son homologue québécois qui évolue dans le champ d'influence du clergé, l'instituteur laïc en France a été un agent actif du pouvoir étatique. C'est lui qu'on investit, à

17 Cf. J.-M. Mayeur, La séparation de l'Église et de l'État (Paris, Julliard, 1966). M. Ozouf, L'École, l'Église et la République, 1871-1914 (A. Colin, 1963). G. Weill, Histoire de l'idée laïque en France au XIXe siècle (Paris, Alcan, 1925). Il y a également l'excellent essai critique d'E. Poulat, Église contre Bourgeoisie (Paris, Casterman, 1977).

18 En ce qui a trait au rapport Église/État en France au niveau de l'école primaire, voir M. Gontard, L'Enseignement primaire en France de la Révolution à la loi Guizot (Paris, Les Belles Lettres, 1959), ainsi que, du même auteur, L'Oeuvre scolaire de la Troisième République, l'enseignement primaire en France de 1876 à 1914 (Paris, Institut Pédagogique National, 1965). 
partir des années 1880 surtout, d'une sorte de missionnariat républicain et laïc auprès de ses élèves ${ }^{19}$. Mais, par-delà l'enfant, c'est manifestement la famille qui est aussi interpellée. Elle constitue de fait le principal destinataire du message transmis à l'enfant, message que ce dernier est chargé à son tour de traduire à ses parents par ses moyens propres. Écoutons à ce sujet les déclarations de Ferdinand Buisson, un homme politique de la IIIe République, qui fut aussi un des apôtres les plus ardents de la laïcité:

Nous ne le dissimulons pas, vous le voyez, notre ambition est que l'école soit aimable afin que l'enfant l'aime et la fasse aimer. C'est sur lui que nous comptons, c'est à ses faibles mains que nous confions le dépôt sacré de nos espérances. Va, petit missionnaire des idées modernes, petit élève de l'école primaire. Au sortir de ton école, montre à tes parents tout ce que tu en rapportes... ils comprendront vite la portée du changement qui s'est fait, ils devineront bien ce que vaut une telle éducation et à qui ils la doivent; et plus d'une fois peut-être...

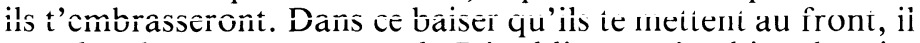
y a plus de promesses pour la République qu'en bien des victoires électorales. ${ }^{20}$

Les résultats de l'offensive étatique au niveau des écoles primaires publiques se révélèrent d'ailleurs à la mesure des efforts qu'investirent dans ce champ les gouvernements successifs, surtout depuis la fin du Second Empire. En effet dès 1877, les statistiques officielles révèlent qu'au niveau primaire - du moins jusqu'à l'âge de 12 ans - près de $80 \%$ des garçons scolarisés le sont dans une école publique, alors que cette proportion baisse jusqu'à $70 \%$ en ce qui a trait aux filles ${ }^{21}$. Ainsi bien avant les lois «fondamentales» promulguées en 1880 et 1881 sous le ministère de Jules Ferry, lois visant à instaurer un système scolaire gratuit, obligatoire et laïc, bien avant les restrictions progressives dont seront l'objet les écoles tenues par les institutions religieuses (dites «écoles congréganistes»), l'État français avait réussi à accaparer une très grande partie du champ éducatif au niveau élémentaire. Il s'y présentera désor-

19 Cf. G. Duveau, Les Instituteurs (Paris, Seuil, 1957). J. Ozouf, «L'Enquête d’opinion en histoire. Un exemple: l'instituteur français, 1900-1914», in Le Mouvement social, (juillet-septembre 1963): 3-22. La marge de manoeuvre accordée à l'instituteur laïc ne semble pas toutefois avoir toujours été en concordance avec l'ampleur de la mission qu'on disait vouloir lui confier. Voir à ce sujet: A. Querien, "L'enseignement», in Recherches, no 23, juin 1976. cit., 397-398.

20 F. Buisson, «Discours à l'association polytechnique, 1883», cité par A. Prost, op.

21 A. Prost, op. cit., Cf. tableau statistique, 100. 
mais comme le principal intervenant auprès des enfants et des familles qui évoluent dans l'orbite de l'école publique.

Par ailleurs dans le secteur secondaire de l'enseignement la présence de l'État en France s'instaure à un rythme différent ${ }^{22}$. Ici le groupe social concerné diffère également: il s'agit en majorité d'enfants et de familles issues de la bourgeoisie et, dans une certaine proportion, de la petite bourgeoisie ${ }^{23}$. C'est d'ailleurs d'une fraction de ces mêmes groupes - qui avaient assuré jusque-là sa clientèle à l'école secondaire privée - qu'émergeront au milieu du 19e siècle les premières pressions pour une prise en charge par l'État d'un enseignement primaire plus poussé, désigné plus tard comme primaire supérieur, complémentaire, puis secondaire. Dans ce dernier champ toutefois l'implantation du pouvoir étatique s'est avérée plus lente et plus difficile. Elle fut confrontée à une concurrence sérieuse de la part du secteur secondaire privé où l'influence cléricale - nous le verroris plus loin - demeura longtemps omniprésente. À ce niveau toutefois, les difficultés éprouvées par l'État français ne vont pas jusqu'à le mettre sur un pied d'égalité avec son homologue québécois. En effet pendant qu'au Québec les collèges classiques occupent une place prépondérante dans le secteur secondaire et collégial, pendant que le niveau universitaire échappe en grande partie, jusqu'au milieu du 20e siècle, à l'ingérence de l'État, ce dernier en France s'est donné, à travers l'Université, un instrument de contrôle remarquable. Un instrument qui fera la preuve de son efficacité en particulier sous la IIIe République ${ }^{24}$. Aussi malgré les énormes privilèges qu'accordait la loi Falloux (1850) à l'enseignement congréganiste - qui recouvre jusqu'en 1880 une entière liberté d'action - l'Université réussira, après cet «entracte» de trois décennies, à réinsérer dans le cadre de sa juridiction l'ensemble du secteur secondaire et universitaire ${ }^{25}$. Sans aller jusqu'à évincer le

22 Sur l'histoire de l'enseignement secondaire en France au 19e siècle, voir la remarquable synthèse de C. Weill, Histoire de l'enseignement secondaire en France (1802-1920) (Paris, Payot, 1921).

23 C'est en effet dans ces classes que se recrutait en majorité la clientèle de ce que l'on a désigné au 19e siècle comme «l'école des notables» (cf. A. Prost, op. cit., chapitre I).

24 Sur l'origine de l'Université en France et sa définition comme un prolongement naturel du pouvoir étatique, voir l'ouvrage d'A. Aulard, Napoléon ler et le monopole universitaire (Paris, A. Colin, 1911). Au sujet d'une synthèse de l'évolution de l'enseignement supérieur en France, voir l'article de T. Zeldin, "Higher Education in France, 1848-1940», in Journal of Contemporary History (nov. 1967): 53-80.

25 Au sujet de l'impact de la loi Falloux sur le développement de l'enseignement congréganiste et sur l'opposition Église/État dans le contexte duquel elle s'inscrit, voir l'excellente thèse de J. Maurain, La politique ecclésiastique du Second Empire (Paris, Alcan, 1930). 
pouvoir religieux de ce domaine, elle permettra à l'État de demeurer présent dans un champ du social oi il était en mesure de rejoindre une fraction importante des enfants et des familles de la bourgeoisie.

\section{Les analogies}

Peut-on, à partir de ce qui précède, conclure à une opposition radicale entre les deux sociétés française et québécoise en ce qui a trait à la structure et à l'évolution du rapport Famille/Église/État dans le champ de l'éducation? Cette opposition a souvent constitué l'arrière-fond des polémiques menées au Québec, à la fin du 19e siècle, relativement à la laïcisation du secteur éducatif en France et à l'éviction totale de l'Église de ce domaine. Or il nous apparaît qu'une telle perspective, sans être totalement fausse, demeure sommaire. Il faut se rappeler cependant qu'elle s'inscrivait dans un contexte dominé par le spectre d'une laicisation possible de l'éducation, dans un Québec ouvert aux arguments des idéologues cléricáúx ein France, un Québec qui se croyait menacé alors par la contagion laïque.

À la lumière d'une lecture plus attentive de l'historiographie française récente, il apparaît aujourd'hui que la situation de l'Église en France à la fin du 19e siècle n'était pas aussi précaire que l'affirmaient la plupart de ses défenseurs ici et outre-mer. En ce qui regarde plus particulièrement le domaine de l'enseignement secondaire privé, le rapport Église/Famille dans la société française est intéressant à analyser de plus près. Il révèle en particulier que la présence du clergé dans ce domaine n'a pas vraiment souffert, du moins jusqu'au début du 20e siècle, des lois laïcisantes des années 1880. Bien au contraire, on constate que l'enseignement secondaire privé, qui était en majorité laïque jusqu'au milieu du 19e siècle, se confessionnalise de plus en plus à partir de cette date. L'historien A. Prost affirme même à son sujet: «De 1865 à 1876, au contraire, il prend une avance réelle, et la Troisième République assiste à son expansion d'autant plus remarquable qu'elle contraste avec la stagnation globale de l'enseignement secondaire. Les mesures violentes de 1880 n'entraînent qu'un recul passager; en 1898 il y a plus d'élèves dans les collèges catholiques que dans les lycées, et nous ne comptons pas les petits séminaires dont pourtant une partie des élèves ne persévère pas dans la carrière ecclésiastique. ${ }^{26}$ Ainsi pour 
l'année 1898, les statistiques officielles du Conseil Supérieur de l'Instruction Publique en France révèlent que les effectifs scolaires dans les collèges catholiques se montent à 67643 élèves alors qu'ils ne sont que 52372 dans les lycées ${ }^{27}$.

Après la trêve qu'instaure la première guerre mondiale dans la question scolaire (c'est alors l'union sacrée entre partisans de la laïcité et cléricaux), après le statu quo qui marque la période d'entre les deux guerres, la lutte reprendra de plus belle au début des années 1940. Elle se soldera pour l'Église par des gains substantiels: en 1940, une loi abroge l'interdiction d'enseigner, imposée aux congréganistes par les lois de 1901 et de 1904. L'année suivante une autre loi sera votée pour permettre aux municipalités de subventionner les «écoles libres» et permettre aux enfants qui fréquentent ces écoles l'accès aux bourses de l'État ${ }^{28}$. C'est d'ailleurs autour de cet aspect financier que s'organiseront une grande partie des débats menés autour de l'école libre. Là encore la question allait se solder par un gain substantiel pour l'enseignement confessionnel puisqu'en 1951 une loi sera votée pour remplacer la subvention étatique aux écoles libres (supprimée en 1945) par une allocation aux parents des élèves qui fréquentent ce type d'institutions ${ }^{29}$.

Face à la question scolaire, la bourgeoisie au pouvoir en France a été, durant plus d'un siècle, manifestement divisée. Le combat autour de la laïcité n'a pas épousé les lignes de démarcation qui séparaient alors les classes sociales en présence ${ }^{30}$. Mais dans ce combat pour maintenir l'intégrité de l'influence cléricale dans le domaine éducatif un aspect retient particulièrement notre attention: l'Église a su, ou a dû, intégrer la famille dans l'ensemble de ses stratégies de lutte. Dans le secteur secondaire et universitaire toutefois il s'agissait plus de la famille bourgeoise que de la famille prolétarienne puisque c'est la première surtout que concernaient les

27 Statistique de l'enseignement secondaire en 1898. Cité par A. Prost, op. cit., 45.

28 Ibid., 472-482. Il est à noter qu'au début du 20 e siècle, «l'école libre» représente le terme le plus courant pour désigner l'école privée en général, et, de façon plus particulière, l'école privée confessionnelle.

29 C'est seulement à partir de 1959 que la loi Debré liera l'octroi de l'aide étatique à la constatation officielle du caractère d'intérêt collectif de l'institution subventionnée et au droit de contrôle exercé par l'État sur cette institution.

30 Au sujet du rapport entre la laïcité et les allégeances politiques et religieuses voir: R. Rémond, "Évolution de la notion de laïcité entre 1919 et 1939", in Cahiers d'histoire, I (1959): 71-87. A. Coutrot et F.-G. Dreyfus, Les Forces religieuses dans la société française (Paris, A. Colin, 1966). 
politiques scolaires à ces deux niveaux ${ }^{31}$. Or l'Église en France a réussi, dans sa pratique autant que dans son discours, à identifier ses intérêts à ceux de la famille dans son ensemble et ceci sans distinction de classes ${ }^{32}$. À l'exemple du schéma qui a prévalu dans la société québécoise à la fin du 19e siècle, le pouvoir religieux s'est présenté au sein de la société française comme le principal défenseur de l'autorité paternelle. Une autorité dite menacée par les prétentions d'un État dont les ambitions démesurées remettait en cause à la fois les prérogatives de l'Église et un certain ordre social lié à l'intégrité du modèle familial qu'elle défendait. Cette perspective s'est traduite dans maintes localités rurales en France, jusqu'au début du 20e siècle, par une alliance efficace entre les familles et le curé de la paroisse contre l'instituteur laïc ${ }^{33}$. Pour dénoncer l'impossible neutralité religieuse de ce dernier, le sénateur M. Jouin, au lendemain des lois laïcisantes de 1881, s'exprime ainsi devant le Parlement:

Par un mot, par un geste ou par un sourire, cet instituteur qui ne croit à rien, sans le vouloir, sans même y mettre de la maüvaise volonté, fera passer sur l'âme de l'enfant, je ne sais quel souffle glacé qui paralysera les efforts de ses parents et ceux du curé. ${ }^{34}$

C'est encore vers la famille que se tournent les pouvoirs religieux vers la fin des années 1900 pour contenir l'ingérence du pouvoir étatique dans le secteur des écoles libres. Les Associations de parents d'élèves de l'enseignement libre (ou A.P.E.L.) qui naîtront alors, prouveront surtout leur efficacité lors des batailles menées en 1948 pour amener l'État à subventionner ce type d'institutions qui sont, pour la très grande majorité, rappelons-le, des écoles confessionnelles.

Dès le milieu du 19e siècle, le rapport Église/Famille dans la société française s'avère également bien articulé en ce qui touche à

31 Il faut préciser toutefois que dans certaines régions du nord-ouest et du sud-ouest de la France, l'adhésion aux perspectives éducatives du clergé s'est manifestée dans l'ensemble des catégories sociales.

32 Voir à ce sujet les écrits sur la question scolaire des principaux idéologues cléricaux en France au cours de la seconde moitié du 19e siècle. On constate qu'en ce qui se rapporte à l'alliance "naturelle» entre l'Église et la famille, la pensée des conservateurs tels Mgr Gaume ou le cardinal Pie, rejoint celle des plus libéraux tels Mgr Dupanloup ou Mgr Maret.

33 Voir J. Ozouf, Nous, les maîtres d'école, autobiographies d'instituteurs de la Belle Époque (Paris, Julliard, 1967).

34 Discours de M. Jouin au Sénat, Journal officiel des débats du 3 juin 1881. Cité par A. Prost, op. cit., 201. 
l'éducation des filles, dans le secteur secondaire en particulier ${ }^{35}$. Or s'il est communément admis que l'école et l'éducation en général sont des courroies de transmission privilégiées dans l'élaboration d'un modèle familial précis, il est clair que l'éducation des filles en constitue une (courroie) plus privilégiée encore. Les pouvoirs étatiques et religieux s'accordent d'ailleurs là-dessus. On sait comment en France l'État a cherché, dès la fin du Second Empire, à organiser et à insérer dans son champ d'influence les institutions d'enseignement secondaire destinées aux filles ${ }^{36}$. Les célèbres cours secondaires publics organisés depuis 1867 par Victor Duruy, alors ministre de l'Instruction publique, en témoignent. Après le succès mitigé de ce type d'enseignement (cours non-inclus dans un programme précis et ne débouchant sur aucun diplôme) une deuxième tentative, plus cohérente et plus fructueuse celle-là, sera menée par le gouvernement de la IIIe République à partir de l'adoption, en 1880, de la loi Camille Sée visant à la création de lycées féminins subventionnés par l'État. Mais dans cette entreprise, comme dans bien d'autres, le pouvoir étatique devra compter avec l'Église, qui avait d'ailleurs sur l'État une avance appréciable dans le secteur de l'éducation féminine. Elle le manifestera à travers l'étendue de la campagne d'opinion menée par les idéologues cléricaux contre l'ingérence du pouvoir civil dans ce domaine. L'État était accusé de chercher à empiéter sur les prérogatives «naturelles» de l’Église, mettant ainsi en péril non seulement l'autorité religieuse et morale de cette dernière mais également la stabilité d'un modèle familial (chrétien) dont l'Église était jusque-là la principale garante ${ }^{37}$.

Il est vrai que si l'hostilité du clergé fut déterminante dans l'échec des cours dont rêvait Victor Duruy, elle ne réussira pas pour autant à freiner l'implantation lente mais progressive des lycées et des collèges de filles sous la IIIe République. Il n'en demeure pas

35 Au niveau de l'éducation secondaire des filles au 19e siècle, c'est encore, rappelons-le, de la famille bourgeoise qu'il s'agit bien plus que de celle des classes populaires. L'impact cependant n'en demeure pas moins important sur le modèle familial véhiculé à travers la société globale.

36 Les ouvrages de synthèse relatifs à l'éducation des filles sont rares. Deux cependant ont été publiés récemment, qui compensent par leur qualité remarquable la carence d'études menées jusqu'ici dans ce domaine. Il s'agit des deux ouvrages de Françoise Mayeur: L'enseignement secondaire des jeunes filles sous la Troisième République (Paris, Presse de la Fondation nationale des sciences politiques, 1977) et L'Éducation des filles en France au XIXe siècle (Paris, Hachette, 1979).

37 On retrouve à ce sujet les arguments les plus significatifs dans les polémiques engagées autour de la question dans Le Monde, L'Univers, mais surtout sous la plume de l'évêque d'Orléans, Mgr Dupanloup. 
moins que ces institutions continuèrent pendant près d'un demisiècle à être soumises à une concurrence sérieuse de la part des couvents et des pensionnats privés (une grande partie de ces derniers calquait sa philosophie et ses programmes sur ceux des couvents) ${ }^{38}$. C'est en tenant compte d'ailleurs de cette concurrence et des principes moraux qui l'alimentaient que l'État écartera pour ses propres établissements le principe de l'école mixte. L'influence de l'Eglise se retrouvera également dans le modèle familial diffusé à travers le programme secondaire offert aux filles. Ce dernier sera élaboré en fonction du rôle futur assigné à la jeune fille au sein de la famille; d'où son caractère moins utilitaire que le programme offert aux garçons; d'où également la place importante qui y sera longtemps faite à l'enseignement ménager ${ }^{39}$.

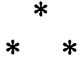

Cette analyse, quelque peu sommaire, ne constitue indéniablement qu'une première approche du vaste champ d'investigation que constituent les rapports Famille/Église/État dans le domaine éducatif. Elle permet de déceler cependant des analogies intéressantes entre les sociétés française et québécoise en ce qui a trait au rôle et à la place de l'Église au sein de ses rapports. Elle nous amène en particulier à nuancer les théories relatives à la constitution de la famille moderne et aux processus et modalités de son intégration dans les réseaux des appareils d'État. La problématique qui sous-tend ces théories évacue trop vite, nous l'avons signalé plus haut, la place et le rôle effectif de l'Église. On constate que même en France, où plus tôt qu'ici l'État s'affirme centralisateur et bureaucratique, l'influence de l'Église dans le champ du social n'a accusé jusqu'au milieu du 20e siècle, qu'un recul progressif, étalé dans le temps, et surtout partiel.

Nous croyons que les analyses socio-historiques relatives à la famille se doivent de tenir compte d'un tel élément et de nuancer, à partir de là, les conclusions sur lesquelles elles débouchent relativement au rapport entre la famille et l'État moderne dans la société capitaliste.

38 A. Prost, op. cit., tableaux statistiques, 205. Aussi F. Mayeur, L'éducation des filles en France au XIXe siècle, chap. VI.

39 Sur les «traits spécifiques» des programmes secondaires féminins, voir l'analyse de F. Mayeur in L'Enseignement secondaire des jeunes filles sous la Troisième République, chap. IV. Nous serons amenés également, dans une prochaine étude, à analyser de façon plus détaillée la philosophie et le contenu des programmes éducatifs féminins en France au cours du siècle dernier en nous plaçant dans une perspective comparative par rapport au Québec. 


\section{Conclusion}

Notre analyse s'est intéressée aux rapports complexes qu'entretiennent l'Église, l'État et la famille dans diverses sociétés capitalistes et, en particulier, en France et au Québec, au cours du 19e siècle et de la première moitié du 20 e siècle. Il importe de souligner que nos questions, nos hypothèses et notre interprétation comparative des faits relatifs à la famille, se limitent strictement aux stades du développement de la société bourgeoise qui se situent dans cette période de l'histoire, en Europe occidentale et en Amérique du Nord. Dans la seconde moitié du 20e siècle, les questions relatives à la famille dans la société capitaliste, soulèvent des problèmes différents de ceux qui constituent la matière de notre recherche. En effet, au cours des dernières décennies, la prise en charge à peu près complète de la famille par l'État, apparaît comme un phénomène caractéristique de l'ensemble des sociétés capitalistes et des sociétés socialistes, y compris les sociétés comme le Québec, les États-Unis, l'Italie, l'Espagne et autres où l'État exerçait relativement peu d'influence sur la famille, dans les périodes précédentes. Dans ces sociétés, cette évolution s'accompagne en priorité, d'une modification du système scolaire ainsi que du système de l'assistance sociale dont la structure et les pratiques s'alignent en quelque sorte sur le modèle du contrôle étatique, lequel représentait, dans les périodes précédentes, un pôle extrême de la typologie des relations triangulaires entre la famille, l'Église et l'État.

Au Québec, c'est vers la fin des années 1950 que l'organisation de la société québécoise dans l'Église, sur la base de la famille, commence à s'affaiblir et que s'impose progressivement la nécessité d'une prise en charge par l'État de l'ensemble des fonctions liées au contrôle de la population. En effet, ces fonctions présentent une telle complexité et exigent la mise en oeuvre de ressources telles que l'Église ne peut désormais y suffire. Ainsi, les tâches d'enregistrement, de régulation et d'encadrement jadis assumées en bonne partie par l'Église, seront entièrement étatisées. Au cours de la période dite de la révolution tranquille, le système scolaire - enjeu majeur de cette révolution - de même que le système des soins de santé et celui de l'assistance sociale, passeront sous l'autorité quasi exclusive de l'État québécois. Ces services seront transformés en fonctions bureaucratiques, leur étatisation provoquant d'ailleurs une croissance extraordinaire des appareils étatiques et para-étatiques. De même, la culture sera «laïcisée» et placée sous la responsabilité de l'État. Désormais, c'est également l'État qui encadrera la famille 
québécoise et c'est vers l'État que celle-ci devra se tourner pour obtenir les ressources et les services dont elle ressent le besoin. Ses «problèmes» deviendront ceux de la famille «moderne»: contraception, divorce, travail des femmes, crise de l'autorité parentale, etc. De même, les moyens et les techniques d'intervention, mis en oeuvre par l'État auprès de la famille s'inspireront des principes qui régissent les politiques familiales de la plupart des autres Etats occidentaux.

On peut toutefois se demander si cette famille occidentale moderne, c'est-à-dire universellement étatisée, n'est pas fondamentalement différente de celle que l'État bourgeois, l'Église et d'autres appareils, ont constituée et reproduite en tant que rouage des sociétés capitalistes du $18 \mathrm{e}, 19 \mathrm{e}$ et du début du $20 \mathrm{e}$ siècles. Il est probable, nous semble-t-il, que la famille subisse actuellement une transformation profonde de sa forme, de ses fonctions et de ses rapports avec les autres appareils de la production, du contrôle et de la reproduction, lesquels aussi se sont profondémentit môdifiés au cours des dernières décennies. Nous ne sommes pas en mesure de démontrer et d'illustrer cette hypothèse dans les limites du présent travail. Nous devons cependant signaler que le discours dominant sur la famille empêche que cette question du changement de l'organisation familiale, soit posée et résolue de manière satisfaisante. En effet, dans le discours des appareils, la famille est, aujourd'hui comme hier, identique à elle-même, c'est-à-dire à son essence. La conception que l'idéologie présente de la famille rejoint, à cet égard, la conception des théories fonctionnalistes que nous avons résumée précédemment: la famille est une institution universelle et éternelle, exerçant des fonctions essentielles à la satisfaction de besoins vitaux de l'humanité. C'est dans cette optique que les politiques de l'État et des autres appareils à l'endroit de la famille sont définies comme un soutien, une suppléance, une assistance à la famille dans l'exercice de ses fonctions essentielles, alors que ces politiques ont imposé progressivement une transformation de la forme de la famille et de ses fonctions. La stratégie et les effets de cette transformation varient d'ailleurs selon les classes sociales et les milieux auxquels elle s'applique. Qu'on pense, entre autres, aux répercussions profondes qu'entraîne l'extension de la période d'encadrement par l'appareil scolaire de la majorité de la population, extension qui se fait tant par le haut (secondaire, collégial, universitaire et éducation permanente) que par le bas (garderie, maternelle, etc). Qu'on pense aussi aux conséquences de la ramification du 
système de l'assistance sociale en général et de sa pénétration dans la vie quotidienne de la quasi-totalité de la population, par le moyen de l'assurance-chômage, de l'assurance-santé, des rentes et pensions, des services sociaux, médicaux, psychiâtriques, conjugaux, des subventions, allocations et aide de toutes sortes. C'est aussi dans les limites et le cadre de cette conception fonctionnaliste de la famille que les revendications et les projets féministes (qui impliquent également un bouleversement de la forme de la famille et de ses fonctions) sont reçus et ré-interprétés par l'État et par les autres pouvoirs en place. Il revient aux chercheurs, en particulier, de cerner et d'analyser, au-delà de ce discours de l'idéologie sur la famille, les modalités et les potentialités de cette nouvelle réorganisation de la famille dans la société capitaliste actuelle.

Par ailleurs, si l'étatisation universelle de la famille a impliqué le retrait des églises (retrait volontaire ou forcé selon les cas), hors des systèmes qui structurent la famille, l'influence idéologique de l'appareil religieux subsiste dans ce champ plus que dans n'importe quel autre secteur de la vie sociale. Comme certains chercheurs contemporains l'ont signalé, l'appareil d'État n'a ni les ressources, ni la légitimité nécessaire pour «produire» de la culture, et en particulier, pour fonder et formuler un discours moral ${ }^{40}$. Or, on peut considérer que dans le champ des relations familiales et de la vie dite privée en général, l'usage d'une morale dont les principes et les règles s'appuient sur une représentation transcendante, supérieure ou extérieure à la raison d'État, s'avère sinon indispensable du moins très profitable. À cet égard, il faudrait se pencher sur les formes actuelles du discours religieux sur la famille et tenter de repérer les nouveaux mécanismes qui peuvent permettre l'inscription fonctionnelle de ce discours dans la stratégie étatiste de réorganisation de la famille. 